evaluate cost-effectiveness studies of PR programmes worldwide.

Methods A systematic review was conducted in accordance with PRISMA guidelines. A thorough literature search strategy was employed across PubMed, the Cost-Effectiveness Analysis Registry (CEA Registry), National Health Service Economic Evaluation Database (NHS EED), Physiotherapy Evidence Database (PEDro) and Google Scholar from inception to October 2019 for studies comparing the cost-effectiveness of PR programmes with that of usual care. Included studies had to meet the Cochrane definition of PR; at minimum, exercise training for at least 4 weeks. Cost-effectiveness measures included cost per quality-adjusted life year (QALY), cost per clinically significant outcome, incremental cost-effectiveness ratio (ICER) and/or cost savings to the healthcare system involved. These findings were then narratively synthesised.

Results 8 studies consisting of 1437 patients were included. Settings for the PR programmes were UK, Ireland, France, Netherlands, Canada and Australia. 7 studies included COPD patients only. 1 study assessing the uncertainties around the cost and outcome found that the cost per QALY was below $£ 17000$, below the willingness to pay threshold suggested by the NICE. Evidence from the studies suggests that PR is cost-effective with savings for the healthcare provider involved.

Conclusion PR is a cost-effective intervention with potential savings for the service providers. Future studies should examine whether cost-effectiveness varies with the age of patients undergoing PR.

\section{THE MANAGEMENT OF BREATHLESSNESS IN A PALLIATIVE CARE INPATIENT UNIT}

Ella Davies, Simon Roughneen, Jessica Lee, Andrew Khodabukus, Sarika Hanchanale. Royal Liverpool University Hospital, University of Liverpool

\subsection{6/spcare-2020-PCC.205}

Background Breathlessness is prevalent in patients at the end of their life and the aetiology is often multifactorial. This symptom is distressing for both patients and their families and can be a significant cause of morbidity. Current evidence based regional guidelines recommend the use of non-pharmacological and pharmacological interventions, of which, modified-release opioids are the mainstay. This audit examines how successfully these guidelines are complied with in an inpatient palliative care setting.

Methods A retrospective case note analysis collated a list of all patients admitted to the academic palliative care unit (APCU) from 1st October to 30th November 2019. Data on breathlessness management was gathered through the trust$\hat{\mathrm{a}} €{ }^{\mathrm{TM}} \mathrm{s}$ electronic documentation system and compared with regional guidelines.

Results Out of 80 patients admitted to the APCU, 32 (40\%) had breathlessness. Of these 32, the most common diagnosis was cancer (not lung primary) at $43.8 \%$, followed by cancer (lung primary) at $21.9 \%$. Around $80 \%$ had a reversible cause of breathlessness, all of which were treated. Non-pharmacological options were offered to $68.8 \%$ of patients, which was a hand-held fan in $31 \%$ of cases. Half of the patients had symptoms of anxiety and of these, $93.8 \%$ were offered anxiolytics. Nebulisers were utilised in 50\% of patients of which $88.2 \%$ were saline. Opioids were suggested for patients who were not already prescribed one. Modifiedrelease morphine and instant-release oxycodone were most commonly used followed by instant-relief morphine. It was common for steroids, oxygen and antibiotics to be used as adjuvant treatment.

Conclusion This audit confirms previous findings that breathlessness is a significant symptom in palliative patients. Based on the current regional guidelines, non-pharmacological interventions and modified-release morphine could be offered to more patients. Further discussion through interactive feedback and education is a priority to comply with current guidelines.

\section{6}

\section{OLANZAPINE FOR THE MANAGEMENT OF DELIRIUM IN THE PALLIATIVE CARE SETTING - A SYSTEMATIC REVIEW OF THE EVIDENCE}

Mairéad Doherty, Feargal Twomey. Milford Care Centre, Limerick

\subsection{6/spcare-2020-PCC.206}

Background Delirium is a neurocognitive syndrome common in palliative care. Although typical antipsychotics are the pharmacological management of choice for distressing symptoms of delirium, the atypical antipsychotic olanzapine may have a role.

Aim To evaluate the evidence for olanzapine, given orally or subcutaneously (sc), in the management of delirium in adults in the palliative care setting.

Methods Electronic databases (Embase, MEDLINE, and PsycINFO) were searched in March 2019 complemented by hand-searching using reference lists and review articles. All studies excluding case reports were included. The primary and secondary outcomes were reduction in delirium severity and toxicity respectively. Relevant studies were summarised and synthesised. Meta-analysis was not performed due to heterogeneity in the studies.

Results Five studies with 244 participants were included in the analysis - one randomised controlled trial, one nonrandomised trial with a control arm, two prospective cohort studies, and one cross-sectional study. All studies were in cancer patients. Delirium severity reduced by an average of 6.93 on the Memorial Delirium Assessment Score at one week, with no statistical difference seen between olanzapine and alternative antipsychotics. Olanzapine did not cause extrapyramidal side effects (EPSE) in any study but did lead to sedation in three studies $(10-30 \%$ of participants affected). One study assessed the safety and efficacy of sc olanzapine - no injection site toxicity was seen but the sample was small, attrition was high, and systemic toxicity was noted. The overall quality of the studies was graded as weak.

Conclusion Olanzapine has weak evidence of comparative effectiveness to other antipsychotics in reducing delirium severity in patients with cancer. A lack of EPSE suggests a potential role in the management of delirium in Parkinson's disease and Lewy body dementia but further studies are required to evaluate this. There is insufficient evidence to support the use of olanzapine sc in this setting. 